\title{
Influence of mid and low paraplegia on cardiorespiratory fitness and energy expenditure
}

\author{
Gary J. Farkas $\mathbb{D}^{1} \cdot$ Phillip S. Gordon ${ }^{1} \cdot$ Ann M. Swartz ${ }^{2} \cdot$ Arthur S. Berg $^{3} \cdot$ David R. Gater $\mathbb{D}^{1}$
}

Received: 8 October 2020 / Revised: 11 November 2020 / Accepted: 13 November 2020

(c) The Author(s), under exclusive licence to International Spinal Cord Society 2020

\begin{abstract}
Study Design Observational, Cross-sectional.

Objective Examine the influence of mid (MP) and low (LP) paraplegia on cardiorespiratory fitness (CRF), energy expenditure (EE), and physical activity levels (PAL), and compare these data to able-bodied (AB) individuals.

Setting Academic medical center.

Methods Persons with MP $\left(n=6\right.$, T6-T8, 83\% male, age: $31 \pm 11$ y, BMI: $\left.24 \pm 7 \mathrm{~kg} / \mathrm{m}^{2}\right)$ and LP $(n=5$; T10-L1, $100 \%$ male, age: $39 \pm 11$ y, BMI: $\left.26 \pm 5 \mathrm{~kg} / \mathrm{m}^{2}\right)$ and $\mathrm{AB}$ controls $\left(n=6\right.$; $67 \%$ male, age: $29 \pm 12 \mathrm{y}$, BMI: $\left.26 \pm 5 \mathrm{~kg} / \mathrm{m}^{2}\right)$ participated. All participants underwent 45-min of arm-crank exercise where CRF and exercise EE were measured. Basal metabolic rate (BMR) was measured, and total daily EE (TDEE) and PAL were estimated.

Results Absolute $\mathrm{VO}_{2 \text { Peak }}$ (MP: $1.6 \pm 0.2$, LP: $1.9 \pm 0.1$, AB: $2.5 \pm 0.7 \mathrm{l} / \mathrm{min}$ ), peak metabolic equivalents (MP: $6.8 \pm 1.3$, LP: $5.7 \pm 0.7, \mathrm{AB}: 8.8 \pm 0.8 \mathrm{METs}$ ), peak power output (MP: $72.9 \pm 11.5$, LP: $86.8 \pm 6.1$, AB: $121.0 \pm 34.8$ Watts), and maximal heart rate (MP: $177.7 \pm 9.8, \mathrm{LP}: 157 \pm 13.6, \mathrm{AB}: 185.2 \pm 8.5 \mathrm{bpm})$ were significantly different between the three groups $(p<$ 0.05 ). BMR and TDEE did not significantly differ between the three groups ( $p>0.05$ ), whereas exercise EE (MP: $7.8 \pm 1.2$, LP: $9.5 \pm 0.7, \mathrm{AB}: 12.4 \pm 3.5 \mathrm{kcal} / \mathrm{min}$ ) and PAL (MP: $1.30 \pm 0.04$, LP: $1.32 \pm 0.04$, AB: $1.43 \pm 0.06$ ) significantly differed $(p<0.05)$. In the $\mathrm{AB}$ group, $33.3 \%$ and $66.7 \%$ were classified as sedentary or having low activity levels, respectively, while all persons with paraplegia were classified as sedentary according to PAL classifications.

Conclusion Individuals with MP and LP have lower CRF, exercise EE, and PALs compared to AB individuals.
\end{abstract}

\section{Introduction}

Maximal or peak oxygen consumption reflects the capacity of body systems to adapt to exercise, and therefore cardiorespiratory health and deconditioning [1]. Individuals living with a spinal cord jury (SCI) are among the most physically deconditioned individuals. The physical deconditioning is even more severe with higher levels of injury and injury completeness [2]. After SCI, cardiorespiratory

Gary J. Farkas

gjf50@med.miami.edu

1 Department of Physical Medicine and Rehabilitation, University of Miami Miller School of Medicine, Miami, FL, USA

2 Department of Kinesiology, University of Wisconsin Milwaukee, Milwaukee, WI, USA

3 Department of Public Health Sciences, Penn State College of Medicine, Hershey, PA, USA health is significantly decreased as a result of injury-induced physical deconditioning, impaired fitness, increased sedentary lifestyle [3], impaired ventilatory capacity, decreased tidal volumes [4], sympathetic nervous systems dysfunction [5], circulatory dysfunction [5-7], adaptive myocardial atrophy [8], impaired cardiac output and stroke volume [3], and altered endometabolic milieu that limits physiological responses to exercise $[9,10]$.

Approximately one quarter of individuals with paraplegia do not achieve a level of oxygen consumption on an arm crank ergometry test sufficient to perform several activities of daily living [11]. Several reports dichotomize persons with paraplegia as above ("high paraplegia") or below T5/6 ("low paraplegia"), or consider all persons with paraplegia as a singular study group. However, SCIs below T5/6 include several thoracic, lumbar, and sacral injury levels that present with specific differences in neurological outcomes and various amounts of somatic and sympathetic dysfunction. There is an increased prevalence of lower motor neuron (LMN) injuries among individuals with and 
below a T9/T10 injury level [12]. LMN injuries lead to skeletal muscle atrophy [12,13], hypotonia, fibrillations in the muscles, and flaccid paralysis in the lower limbs [12]. LMN injuries further prevent the use of electrical stimulation therapies to reverse the atrophy and increase energy expenditure (EE) [14, 15]. Somatic denervation of the thoracoabdominal muscles results in the successive level of injury-dependent loss of core/trunk stabilizers, muscles of respiration, and restrictive pulmonary disease [4]. The sympathetic nervous system is also affected. Sympathetic innervation of the splanchnic vascular bed of the foregut is mainly derived from the greater (thoracic) splanchnic nerve at the levels of T5-T9/10 [16], while sympathetic innervation of the lower limb is derived from neurons in the lower thoracic (T10-T12) and upper lumbar (L1, L2) spinal cord segments [17]. The loss of sympathetic innervation at or below the T9/T10 spinal level to a large portion of the gut and the lower limb comprises a significant loss in the regulation of blood pressure. The paucity of literature evaluating physiologic responses to exercise in mid (MP; T5/6T8/9) and low (LP; T9 and below) paraplegia is alarming given that paraplegia accounts for nearly $40 \%$ of SCI $[18,19]$.

Total daily energy expenditure (TDEE) is the sum of the thermic effect of food, the thermic effect of physical activity/exercise, and basal metabolic rate (BMR) [20]. The impact of an SCI on EE remains relatively controversial because BMR considerably varies based on the influences of sex, injury completeness, level of injury, and body habitus [21-23]. A recent systemic review by Farkas et al. [21], reported that close to $70 \%$ of the studies evaluating the influence of level of injury on metabolic rate fail to report a significant difference between individuals with tetraplegia and paraplegia. This may be a consequence of including a heterogenous sample of thoracic, lumbar, and/or sacral level of injuries and injury completeness.

Exercise EE is a key consideration as it is the most variable component of TDEE [20, 21, 24]. Because TDEE depends on activity level, taking into account the amount of physical activity/exercise is essential. The level of physical activity is commonly described by the World Health Organization as the ratio of TDEE to BMR (TDEE/BMR). This ratio is known as the physical activity level (PAL). PALs are a universally accepted way of expressing EE that account for body composition [25]. Nightingale et al. [26] reported a PAL of 1.20 to 1.85 in persons with chronic T1-L4 paraplegia representing a range of $65 \%$ among study participants. To date, no studies have compared PALs in persons with MP and LP.

The characterization of the cardiorespiratory capacities and $\mathrm{EE}$ of both persons with tetraplegia and high paraplegia has been previously studied, but to the authors' knowledge, no studies have investigated the effects of mid and low levels of paraplegia on these parameters. The purpose of this study was to examine the influence of MP and LP on cardiorespiratory fitness (CRF), EE, and PAL, as well as compare these data to able-bodied $(\mathrm{AB})$ individuals.

\section{Methods}

\section{Protocol overview}

All participants had a complete history, physical examination, and completed a graded maximal exercise test at the host institution. Once cleared for participation, participants were scheduled to begin the study within two weeks of the physical exam. The study schedule consisted of a three-day, two-night stay at the General Clinical Research Center (CRC). On the first day, participants reported to the testing laboratory for an assessment of body composition and to be admitted to the CRC. The following morning, BMR was measured. On the third morning of the participants' stay, a 45-min moderateintensity exercise bout was completed and participants were then discharged from the CRC. Participants were provided lunch and dinner during their stay at the CRC.

\section{Participants and physical exam}

All participants were recruited from the host institution over the course of a year and a half. Inclusion criteria were male and female participants between the ages of 18 and 65 years (with maximum age chosen to avoid any confounding effects of age on body composition) with chronic ( $\geq$ one-year post-injury) motor complete paraplegia (American Spinal Injury Association Impairment Scale (AIS) A and B) [27]. In addition, healthy $\mathrm{AB}$, self-identified inactive adults were recruited for participation in the study. Exclusion criteria included individuals in an exercise program within the past three months; smokers; individuals with excessive alcohol consumption ( $\geq 2$ drinks/day); individuals with known cardiovascular disease, diabetes (either type), hypothyroidism, and/or renal disease; uncontrolled autonomic dysreflexia (cases unresolved by removing the noxious stimulation); recent venous thromboembolism; pressure injuries (>grade II); or heterotopic ossification involving the upper limbs. This study was approved by the Institutional Review Board at the host institution and all participants completed informed consent prior to participation in the study.

All participants with SCI underwent a complete physical examination by a board-certified Spinal Cord Medicine Physiatrist prior to participation. The physical examination included a collection of demographic information (age, sex, level of injury, and time since injury), a medical history, AIS examination [27], 12-lead resting electrocardiogram, and resting blood pressure. 


\section{Graded maximal exercise test}

Participants underwent a graded maximal exercise test on an arm crank ergometer (ACE) (Monark 881 Arm Crank Ergometers) with a TrueMax 2400 (ParvoMedics, Salt Lake City, Utah) metabolic measurement system. This allowed for the classification of results in relation to cardiovascular conditioning, provided exercise intensity during the 45-min exercise bout, and screened for potential contraindications for participation in the submaximal exercise bout. A standard, discontinuous ACE protocol was used with an initial resistance of 36 watts and a metronome-guided crank rate of 50 revolutions per minute. A warm-up stage of three minutes was used at this work rate, with subsequent threeminute stages of 12-watt increments used until exhaustion. Peak oxygen consumption $\left(\mathrm{VO}_{2 \text { Peak }}\right)$, respiratory exchange ratio (RER), metabolic equivalents (METs; calculated using $3.5 \mathrm{~mL}$ of oxygen per kilogram of bodyweight per minute), and power output were recorded. Blood pressure and rate of perceived exertion were repeatedly monitored every ten minutes throughout the test, while heart rate (HR) as assessed through a 12-lead II electrocardiogram (Quinton Q710 ECG system, Milwaukee, Wisconsin) was continuously observed. All participants with paraplegia were tested in their own stabilized wheelchair with appropriate seating, truncal stability, leg wraps, abdominal binder, and protective hand mitts that were secured to the ACE pedals.

\section{Body composition}

Fat mass $(\mathrm{FM})$, total percent body fat (\%BF), fat-free mass (FFM), lean body mass (LBM), bone mineral content $(\mathrm{BMC})$, and bone mineral density (BMD) were measured using dual x-ray absorptiometry (DXA) according to previously published methods [22]. The scans were performed after lying supine for a minimum of 20 minutes to reduce fluid shift. All scans were performed using a Lunar Prodigy Advance (Lunar Inc., Madison, Wisconsin), and were performed and analyzed by a certified DXA operator using Lunar software (version 10.5).

\section{The submaximal exercise session}

Participants in all three groups completed a single, highintensity bout of supervised exercise. The exercise bout consisted of three 15-min bouts of ACE at $\sim 75 \% \mathrm{VO}_{2}$, monitored by HR, for a total of 45 minutes of aerobic exercise. This load was chosen for testing of persons with paraplegia because oxygen consumption is dependent on level of SCI [3]. Each 15-min bout of activity was interspersed with two to three minutes of rest. $\mathrm{VO}_{2 \text { Peak }}$, RER, peak METs, and peak power output were recorded. Peak was defined as the highest observed value during the submaximal exercise test [28].
HR, blood pressure, and rating of perceived exertion were monitored throughout the test. HR was monitored and recorded in 60-second intervals using the Polar Vantage NV belt (Polar Inc., Finland) that was attached to an elastic strap placed around the xiphoid process of the sternum of the participant. Blood pressure was recorded by the auscultatory technique using a sphygmomanometer and a stethoscope before the exercise bout, after each 15-minute exercise stage, and after completion of the exercise bout. Rating of perceived exertion was obtained using the Borg 6-20 category scale. This exercise bout occurred only once during the study.

\section{Energy expenditure}

Basal metabolic rate. Following an overnight fast for 10-12 h, BMR was measured according to previously published methods [22]. BMR measurements began at 6:00 AM. Participants were in a dark room in a supine position for 20 minutes to achieve a resting steady state. During this time, BMR was measured using indirect calorimetry with Deltatrac Metabolic Monitor (Datex-Ohmeda, Helsinki, Finland) and a canopy that covered the head and neck.

Total daily energy expenditure. TDEE was estimated according to the Farkas et al. SCI-specific prediction equation as the product of BMR and 1.15 for persons with paraplegia [22]. For $A B$ individuals, TDEE was estimated using the product of BMR and 1.2 according to Long et al. [29].

Exercise energy expenditure. Total exercise energy expenditure $\left(\mathrm{EEE}_{\text {Total }}\right)$ and exercise energy expenditure per minute $\left(\mathrm{EEE}_{\text {Minute }}\right)$ were determined during the submaximal exercise session. EE during the exercise bout was calculated using stochiometric equations assuming that urinary nitrogen excretion was negligible due to the participants being diet and body mass stable and not having renal diseases as previously described [30, 31]:

\section{Physical activity levels}

PAL was determined as the ratio of TDEE to BMR. TDEE for PAL was determined by summing the BMR, the thermic effect of physical activity as determined by the $\mathrm{EEE}_{\text {Total }}$, and the thermic effect of food. The thermic effect of food was predicted as $8 \%$ of TDEE for the $\mathrm{AB}$ controls and $5 \%$ for persons with SCI according to previously published literature [21, 32]. PAL classifications were defined according to Ohkawara et al. [33]. The authors classified a PAL of $1.0-1.39$ as sedentary activity, $1.4-1.59$ as low activity, $1.6-1.89$ as active, and 1.9-2.49 as very active [33].

\section{Data and statistical analysis}

Individuals with paraplegia were subdivided into MP and LP using T9 as the demarcation point [12, 34]. T9 was 
chosen given the increased prevalence of LMN injuries below T9/T10 [12], sympathetic innervation of the splanchnic vascular bed ends at T9/10 [16], and sympathetic innervation of the lower limb begins at T10 [17].

Absolute $\mathrm{VO}_{2 \text { Peak }}$ (in $\mathrm{L} / \mathrm{min}$ ) was used to determine $\mathrm{VO}_{2 \text { Peak }}$ relative to bodyweight ( $\mathrm{mL} / \mathrm{kg}$ of bodyweight $/ \mathrm{min}$ ), and DXA-assessed LBM (mL/kg of LBM/min) and FFM $(\mathrm{mL} / \mathrm{kg}$ of $\mathrm{FFM} / \mathrm{min})$. All data were visually checked for normality with histograms and with the Shapiro-Wilk test of normality. An independent sample $t$-test was used to evaluate time since injury between persons with MP and LP. Comparisons among the groups with paraplegia and the $\mathrm{AB}$ controls were completed using one-way analysis of variance (ANOVA) with Tukey's post hoc test when significance was $<0.05$. The level of significance was set at alpha $<5 \%$. All analyses were performed using SPSS 26.0 (IBM, Armonk, New York).

\section{Results}

\section{Demographics and body composition}

Table 1 presents demographic and body composition characteristics. Time since injury ranged from 1.1 to 16.3 and 4.0 to 19.2 years post-injury in persons with MP and LP, respectively. No significant differences in demographic parameters were observed between individuals with MP and LP and the AB control group $(p>0.05) . \%$ $\mathrm{BF}, \mathrm{FM}$, and BMD were significantly different between the groups ( $p \leq 0.047$; Table 1). Post hoc analysis revealed $\% \mathrm{BF}$ and FM were significantly greater in persons with LP compared to persons with MP and the AB controls ( $p \leq$ 0.012). There were no significant differences in percent body fat and FM between individuals with MP and $A B$ controls. BMD was significantly lower in persons with MP relative to $\mathrm{AB}$ controls $(p=0.042)$. All other body composition variables were not significantly different $(p>$ 0.05; Table 1).

\section{Cardiorespiratory fitness}

Figure 1 presents peak oxygen consumption in persons with MP and LP and the $\mathrm{AB}$ controls. Absolute $\mathrm{VO}_{2 \text { Peak }}$ (Fig. 1a; MP: $1.55 \pm 0.23$, LP: $1.89 \pm 0.14$, and $\mathrm{AB}$ : $2.46 \pm 0.69 \mathrm{~L} / \mathrm{min}, p=0.011)$ and $\mathrm{VO}_{2 \text { Peak }}$ relative to bodyweight (Fig. 1b; MP: $23.90 \pm 4.73$, LP: $20.48 \pm 1.96$, and $\mathrm{AB}: 30.80 \pm 2.85 \mathrm{~mL} / \mathrm{kg} \mathrm{BW} / \mathrm{min}, p=0.001)$, LBM (Fig. 1c; MP: $30.73 \pm 4.55$, LP: $34.4 \pm 5.55$, and $\mathrm{AB}$ : $39.65 \pm 2.96 \mathrm{~mL} / \mathrm{kg} \mathrm{LBM} / \mathrm{min}, \quad p=0.012$ ), and FFM (Fig. 1d; MP: $29.01 \pm 4.16$, LP: $32.43 \pm 5.30$, and $\mathrm{AB}$ : $37.41 \pm 2.89 \mathrm{~mL} / \mathrm{kg} \mathrm{FFM} / \mathrm{min}, p=0.012$ ) were significantly different across the three groups. Tukey's post hoc analysis demonstrated a significantly higher $\mathrm{VO}_{2 \text { Peak }}$ relative to bodyweight in $\mathrm{AB}$ controls compared to both persons with MP $(p=0.010)$ and LP $(p=0.001)$, while no significant differences were found between MP and LP $(p=0.266)$. Persons with MP had a significantly lower absolute $\mathrm{VO}_{2 \text { Peak }}(p=0.009)$ and $\mathrm{VO}_{2 \text { Peak }}$ relative to LBM $(p=0.009)$ and FFM $(p=0.009)$ compared to AB controls. No significant differences were found between LP and both AB and MP $(p>0.05)$. Peak METs and peak power output was significantly different across MP, LP, and the AB controls $(p \leq 0.006)$. Tukey's post hoc analysis demonstrated a significantly higher peak METs in AB controls compared to both persons with MP and LP ( $p \leq$ $0.010)$, while no significant differences were found between MP and LP ( $p>0.05$; Table 2). Peak power output was significantly greater in the $\mathrm{AB}$ controls compared to persons with MP $(p=0.006)$, while it approached a significant difference between the $\mathrm{AB}$ controls compared to persons with LP $(p=0.056)$. Maximal HR (MHR) was significantly different across the three groups $(p=0.002)$ with post hoc analysis revealing a significantly lower rate in persons with LP compared to the two other groups ( $p \leq$ 0.016). No differences were found between the AB controls and persons with MP $(p>0.05)$. All other CRF variables were not significantly different $(p>0.05$; Table 2$)$.

\section{Energy expenditure}

EE data are shown in Table 3. BMR and TDEE did not significantly differ between persons with MP, LP, and the $\mathrm{AB}$ controls $(p=0.432$ and $p=0.352$, respectively). $\mathrm{EEE}_{\text {Total }}$ and $\mathrm{EEE}_{\text {Minute }}$ during the submaximal exercise test were significantly different across the groups $(p=0.011$ for both). Tukey's post hoc analysis showed that the AB controls expended a significantly greater amount of energy compared to MP $(p<0.0009)$, whereas there were no differences noted between the LP and the AB controls $(p=0.121)$ and MP $(p=0.444)$. No other variables were significantly different $(p>0.05$; Table 3$)$.

\section{Physical activity levels}

PALs were significantly different between persons with MP $(1.30 \pm 0.04), \mathrm{LP}(1.32 \pm 0.04)$, and the AB controls (1.43 \pm $0.06)(p=0.001)$. Post hoc analysis determined a significantly greater PAL in the $\mathrm{AB}$ controls compared to the MP $(p=0.001)$ and LP $(p=0.005)$. No significant differences were found between MP and LP $(p=0.758)$. Using the classification system by Ohkawara et al. [33], 33.3 and $66.7 \%$ of the $\mathrm{AB}$ controls were classified as sedentary or having low activity levels, respectively. In persons with paraplegia, $100 \%$ of the participants, regardless of their MP or LP injury level, were classified as sedentary. 
Table 1 Demographic and body composition characteristics of study participants.

\begin{tabular}{lllll}
\hline & Mid Paraplegia & Low Paraplegia & $\begin{array}{l}\text { Able-bodied } \\
\text { Controls }\end{array}$ & $p$-value \\
\hline Sample size $(n)$ & 6 & 5 & 6 & 0.943 \\
Age & $31.42(11.33)$ & $38.80(11.29)$ & $28.74(11.92)$ & 0.363 \\
Gender $(\mathrm{M} / \mathrm{F})$ & $5 / 1$ & $5 / 0$ & $4 / 2$ & 0.931 \\
Body mass $(\mathrm{kg})$ & $67.93(20.68)$ & $93.00(12.90)$ & $79.42(20.19)$ & 0.120 \\
Height $(\mathrm{cm})$ & $169.92(11.68)$ & $176.70(7.50)$ & $175.00(7.16)$ & 0.449 \\
Body mass index $\left(\mathrm{kg} / \mathrm{m}^{2}\right)$ & $23.54(6.83)$ & $29.87(4.56)$ & $25.70(5.32)$ & 0.218 \\
Time since injury $(\mathrm{y})$ & $4.98(5.82)$ & $10.13(5.73)$ & $\mathrm{NA}$ & 0.175 \\
AIS $(\mathrm{A} / \mathrm{B})$ & $5 / 1$ & $5 / 0$ & $\mathrm{NA}$ & $\mathrm{NA}$ \\
Level of Injury & $\mathrm{T} 6-\mathrm{T} 8$ & $\mathrm{~T} 10-\mathrm{L} 1$ & $\mathrm{NA}$ & $\mathrm{NA}$ \\
Body fat $(\%)$ & $26.94(7.36)$ & $42.02(7.80)$ & $23.85(5.99)$ & $0.002^{\mathrm{a}}$ \\
Fat mass $(\mathrm{kg})$ & $16.63(12.73)$ & $33.53(7.73)$ & $14.06(6.46)$ & $0.009^{\mathrm{b}}$ \\
BMD $\left(\mathrm{g} / \mathrm{cm}^{2}\right)$ & $1.19(0.07)$ & $1.24(0.12)$ & $1.36(0.12)$ & $0.047^{\mathrm{c}}$ \\
FFM $(\mathrm{kg})$ & $54.08(10.05)$ & $59.47(11.22)$ & $65.36(16.52)$ & 0.352 \\
LBM $(\mathrm{kg})$ & $51.25(9.77)$ & $56.02(10.75)$ & $61.71(15.81)$ & 0.375 \\
\hline
\end{tabular}

Data are presented as mean (SD).

AIS American Spinal Injury Association impairment scale, $B M C$ bone mineral content, $B M D$ bone mineral density, FFM fat-free mass, $L B M$ lean body mass.

aPost hoc analysis: $\mathrm{AB}$ vs. MP, $p=0.752$; $\mathrm{AB}$ vs. $\mathrm{LP}, p=0.002$; MP vs. $\mathrm{LP}, p=0.012$.

${ }^{\mathrm{b}}$ Post hoc analysis: AB vs. MP, $p=0.890$; AB vs. LP, $p=0.010$; MP vs. LP, $p=0.030$.

${ }^{c}$ Post hoc analysis: $\mathrm{AB}$ vs. MP, $p=0.042$; $\mathrm{AB}$ vs. LP, $p=0.200$; MP vs. LP, $p=0.732$. a.

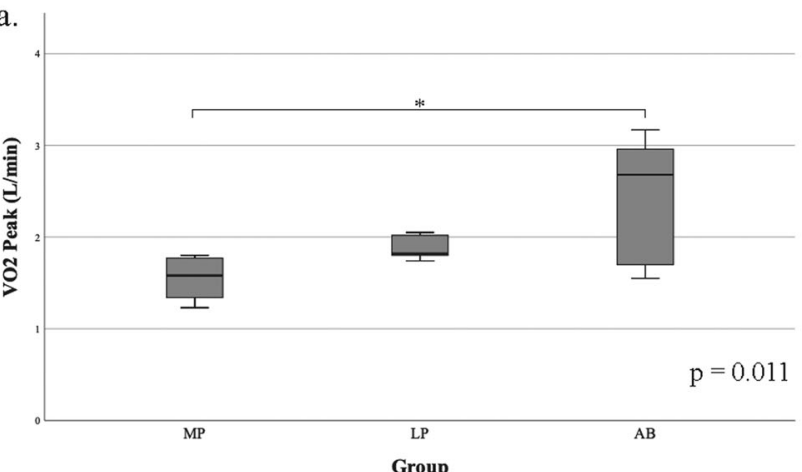

c.

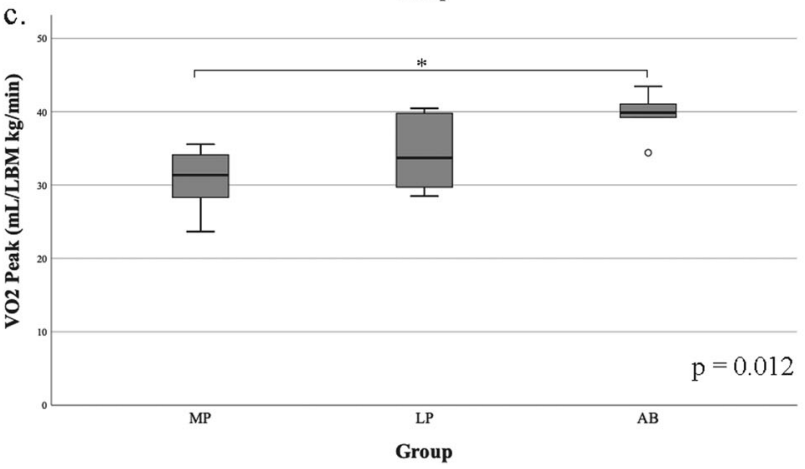

Fig. 1 Peak oxygen consumption $\left(\mathrm{VO}_{2 \text { Peak }}\right)$ in persons with mid paraplegia $(\mathrm{MP}, n=6)$, low paraplegia $(\mathrm{LP}, n=5)$, and the ablebodied controls $(\mathrm{AB} ; \boldsymbol{n}=\mathbf{6})$. Absolute $\mathrm{VO}_{2 \text { Peak }}(\mathbf{a})$ and adjusted $\mathrm{VO}_{2 \text { Peak }}$ to body weight (BW) (b), adjusted $\mathrm{VO}_{2 \text { Peak }}$ to lean body mass $(\mathrm{LBM})(\mathbf{c})$, and adjusted $\mathrm{VO}_{2 \text { Peak }}$ to fat-free mass (FFM) (d) by study b.
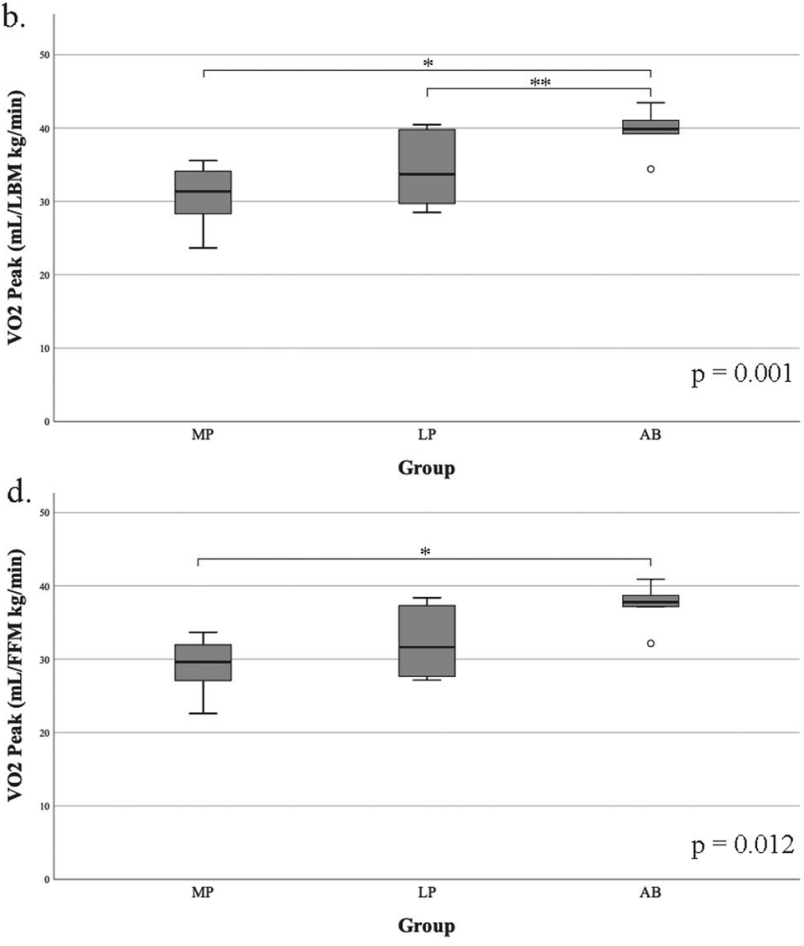

group. One-way ANOVA $p$-values are presented on the bottom right of each panel. *Significant different between MP and AB by Tukey's post hoc, $p \leq 0.009$; **Significant different between $\mathrm{LP}$ and $\mathrm{AB}$ by Tukey's post hoc, $p \leq 0.01$. 
Table 2 Cardiorespiratory fitness in persons with mid (MP) and low (LP) paraplegia and the able-bodied (AB) controls.

\begin{tabular}{|c|c|c|c|c|c|c|c|}
\hline & \multicolumn{4}{|c|}{ One-way analysis of variance } & \multicolumn{3}{|c|}{ Tukey's post hoc comparisons } \\
\hline & $\mathrm{MP}(n=6)$ & $\mathrm{LP}(n=5)$ & $\mathrm{AB}(n=6)$ & $p$-value & $\mathrm{AB}$ vs. MP & $\mathrm{AB}$ vs. $\mathrm{LP}$ & MP vs. LP \\
\hline Respiratory exchange ratio & $1.19(0.12)$ & $1.18(0.09)$ & $1.08(0.12)$ & 0.175 & NS & & \\
\hline $\begin{array}{l}\text { Peak metabolic equivalents } \\
\text { (METs) }\end{array}$ & $6.83(1.33)$ & $5.74(0.66)$ & $8.82(0.82)$ & 0.001 & 0.010 & 0.001 & 0.201 \\
\hline Peak power output (Watts) & $72.93(11.49)$ & $86.76(6.05)$ & $121.00(34.84)$ & 0.006 & 0.006 & 0.056 & 0.570 \\
\hline Resting heart rate (bpm) & $84.17(20.31)$ & $73.60(11.26)$ & $65(9.42)$ & 0.112 & NS & & \\
\hline Maximum heart rate (bpm) & $177.67(9.83)$ & $157.00(13.57)$ & $185.17(8.50)$ & 0.002 & 0.460 & 0.002 & 0.016 \\
\hline Rate of Perceived Exertion & $19.17(1.33)$ & $19.75(0.50)$ & $18.67(2.34)$ & 0.618 & NS & & \\
\hline
\end{tabular}

Data are presented as mean (SD).

$B W$ bodyweight, FFM fat-free mass, $L B M$ lean body mass, $N S$ not significant.

\section{Discussion}

The purpose of this paper was to examine the influence of MP and LP on CRF, EE, and PALs, as well as compare these data to $\mathrm{AB}$ individuals. Data from this study demonstrate that persons with MP and LP have a significantly lower cardiorespiratory response to exercise, and persons with MP have a significantly lower exercise EE compared to $\mathrm{AB}$ controls. We also demonstrated significantly lower PALs in both groups with paraplegia compared to the $\mathrm{AB}$ controls.

\section{Cardiorespiratory fitness}

Cardiorespiratory responses were significantly lower for persons with MP and LP compared to AB controls. Specifically, we demonstrated a significantly reduced $\mathrm{VO}_{2 \text { peak }}$ relative to bodyweight and peak METs in persons with MP and $\mathrm{LP}$ compared to the $\mathrm{AB}$ controls, but absolute $\mathrm{VO}_{2 \text { peak }}$, $\mathrm{VO}_{2 \text { peak }}$ relative to LBM and FFM, and peak power output were significantly lower in persons with MP compared to the controls. Similarly, Holmlund et al. [35] reported that persons with higher level of injuries had levels of oxygen consumption $\sim 50 \%$ lower than individuals with paraplegia during exercise activities. As opposed to $\mathrm{AB}$ individuals, persons with SCI have significant lower limb venous pooling due to paralyzed lower limb muscle pumps and inferior vena cava dilation due to sympathetic blunting limiting preload during exercise. However, individuals with higher levels of injury (e.g., MP) have greater paralysis of segmentally innervated muscles, such as intercostal (e.g., external, internal, and innermost), abdominal (e.g., rectus abdominis and external and internal abdominal oblique), intrinsic back (e.g., iliocostalis and longissimus), and levatores costarum muscles that act as the accessory muscles of respiration during exercise [36]. The greater loss of active muscle mass in those with MP likely explains the significantly reduced absolute $\mathrm{VO}_{2 \text { Peak }}$ and peak power output during exercise compared to those with LP and the $\mathrm{AB}$ controls. This is because both persons with $\mathrm{LP}$ and $\mathrm{AB}$ controls would have more active muscle mass to use for stabilization and as a fulcrum to increase their maximal performance. In the present study, although FFM and LBM were not significantly different between both groups with paraplegia and the $\mathrm{AB}$ controls, LBM was nearly $19 \%$ lower in persons with MP and 10\% lower in persons with LP when compared to the AB controls. Therefore, it is reasonable to conclude that the difference in lean mass (i.e., metabolically active tissue), while nonsignificant, may explain the lower CRF during exercise in MP compared to the $\mathrm{AB}$ controls and LP.

The significantly higher MHR in those with MP compared to persons with LP can be explained by a greater circulator hypokinesis in the former group $[8,37]$. The circulator hypokinesis caused by venous insufficiency and blood pooling below the level of injury would result in a reduced stroke volume and a compensatory tachycardia that stems from an enhanced sympathetic activity. The enhanced sympathetic activity would occur because of the intact sympathetic innervation of the cardiac myocardium in persons with MP [38-40]. At higher exercise intensities, as in this study, the greater sympathetic activity in persons with MP may explain the higher MHR, while those with LP may have a milder form of circulator hypokinesis that is not compensated by an increase in heart rate through sympathetic activity. Healthcare professions may consider providing elastic compression stockings and an abdominal binder for persons with MP to mitigate the effects of the circulatory hypokinesis.

\section{Energy expenditure}

When compared with the $\mathrm{AB}$ individuals, exercise $\mathrm{EE}$ in persons with $\mathrm{SCI}$ is reduced with values representing 30 to $75 \%$ of $\mathrm{AB}$ values [41]. In the present study, exercise $\mathrm{EE}$ at $75 \% \mathrm{VO}_{2 \text { peak }}$ was $63 \%$ of the $\mathrm{AB}$ controls for persons with 
MP and $77 \%$ for LP. The difference in exercise EE may stem from a difference in the absolute intensity participants used during the session. Similar to the present study's findings, Gass and Camp [18] reported an EE of $9 \mathrm{kcal} / \mathrm{min}$ for persons with T10-L3 paraplegia performing wheelchair exercise at 60 to $65 \% \mathrm{VO}_{2 \text { peak }}$. Abel et al. [42] reported the physiological responses to hand biking during a marathon competition in an SCI athlete with a T4 level of injury. The EE was $7.7 \mathrm{kcal} / \mathrm{min}$, close to persons with MP in the present study, but below that of LP [42]. Campbell et al. [43]. examined 12 wheelchair athletes with various levels of paraplegia and reported an expended $5.2 \mathrm{kcal} / \mathrm{min}$ at $70 \%$ $\mathrm{VO}_{2 \text { Peak }}$ during a 60-minute wheelchair exercise session. Similarly, Price and Campbell [44] observed an exercise EE of $6.4 \mathrm{kcal} / \mathrm{min}$ at $60 \% \mathrm{VO}_{2 \text { Peak }}$ in wheelchair athletes with SCI exercising for $60 \mathrm{~min}$. In another study by Price and Campbell $[45,46]$, the authors reported an exercise EE of 6.5 and $6.6 \mathrm{kcal} / \mathrm{min}$ in 10 persons with SCI at $80 \%$ peak heart rate and $60 \% \mathrm{VO}_{2 \text { Peak }}$, respectively. Kressler et al. [47] reported that 12 people with chronic paraplegia under $\mathrm{T} 1$ level of injury underwent a multistage graded exercise test to volitional exhaustion and expended 2.5, 2.8, 3.5, 4.7, and $11.1 \mathrm{kcal} / \mathrm{min}$ at five different stages corresponding with low to high power output. Several of these values are below the current study's finding because the participates in the aforementioned studies were from heterogenous study groups, including persons with T3/4 to L1 paraplegia and a single individual with $\mathrm{C} 6 / 7$ tetraplegia, individuals that had traumatic SCI or spina bifida, SCI athletes, and/or performed exercise at various intensities.

\section{Physical activities levels}

Persons with SCI have frequently been placed on the lowest end of the human fitness continuum, even compared to sedentary persons without SCI [48, 49]. The majority ( $67 \%)$ of the $\mathrm{AB}$ controls in the present study were classified as having low activity levels while all the participants in the paraplegia groups, regardless of the level of injury, were sedentary. Mean PAL of persons with MP (1.30 \pm $0.04)$ and LP $(1.32 \pm 0.04)$ were significantly lower than the $\mathrm{AB}$ controls $(1.43 \pm 0.06)$ in the present study and what is reported in $\mathrm{AB}$ adults in the general population (1.64-1.85) [50]. Buchhloz et al. [23] reported a mean PAL of 1.6 in chronic paraplegia, which was indicative of low activity according to the Ohkawara et al. criteria [25, 33]. Nightingale et al. [26, 51] reported a mean PAL of 1.4 among 33 persons with motor complete T1-L4 paraplegia. A PAL of 1.4 is considered as a low PAL according to Ohkawara et al. criteria [25, 33]. While both Buchhloz et al. [23] and Nightingale [51] evaluated chronic paraplegia in their studies, levels of injury included high, mid, and low paraplegia, which could potentially confound the true PAL 
value. Nonetheless, $\mathrm{AB}$ individuals likely have a greater PAL capacity because of a greater whole-body lean mass compared to persons with paraplegia as persons with motor complete paraplegia cannot use their lower trunks and/or lower limbs in daily activity.

TDEE and BMR cannot always be measured for the determination of PAL. However, quantification of TDEE can occur through estimation using the correction factor developed by Farkas et al. [22], and BMR can be estimated either the Chun et al. [52], or Nightingale and Gorgey prediction equations [53]. These quantities can then be used to determine PAL and quantify the amount of activity or sedentary behavior in persons with SCI. PAL presents an easy method to quantify and classify sedentary behavior in persons with SCI and future research should further study PALs in individuals with tetraplegia $(\geq \mathrm{C} 8)$ and high (T1-T5/ $6)$, mid T6-T8/9), and low ( $\leq \mathrm{T} 9)$ paraplegia.

\section{Limitations}

This study is not without limitations. Small sample size was present in both groups with paraplegia and the $\mathrm{AB}$ controls, creating the opportunity of a type 2 error. We did not include individuals with high paraplegia or tetraplegia, although several articles have previously investigated the influence of higher levels of injury on body composition and metabolic responses to exercise [22, 35, 52-57]. TDEE was predicted and not measured, which could result in a nonsignificant overestimation of the TDEE [21]. However, the correction factor used to estimate TDEE is SCI-specific, thereby limiting the overestimation [22]. Because PAL is calculated as the ratio of TDEE to BMR, the calculation is influenced by the inherent association of BMR in the estimate of TDEE. Therefore, PAL may not be as accurate as if TDEE were measured using a respiratory chamber. Future research should assess TDEE and BMR in a respiratory chamber and determine a PAL that is more precise for persons with MP and LP.

\section{Conclusion}

In conclusion, $\mathrm{CRF}$, exercise EE, and PALs in persons with MP and LP were lower than for AB controls. These data emphasize that persons with MP and LP are at the end of the activity spectrum and help explain the high incidence of obesity within the SCI population, even at lower levels of injury. The evidence from the present study highlights the need for persons with paraplegia to engage in exercise at an increased frequency, duration, and/or intensity to increase exercise EE, increase metabolic rate, and reduce sedentary behavior. It is likely individuals with MP and LP need to engage in increased physical activity/exercise to achieve a PAL $\geq 1.8$ to mitigate the effects of post-SCI changes in body composition, sedentary behavior, and cardiometabolic risk. Future studies should consider tailoring exercise programs prescribed on the basis of high (T1-T5/6), mid (T7$\mathrm{T} 8 / 9$ ), and low (T9/10 and below) paraplegia given the injury level-specific responses to exercise.

\section{Data archiving/availability}

The dataset generated and/or analyzed during the current study are available from the corresponding author on reasonable request, given approval is provided by University's Institutional Review Board.

Acknowledgements This work was supported by the Paralyzed Veterans of America Research Foundation grant \#2256.

\section{Compliance with ethical standards}

Conflict of interest The authors declare that they have no conflict of interest.

Publisher's note Springer Nature remains neutral with regard to jurisdictional claims in published maps and institutional affiliations.

\section{References}

1. Saltin B, Astrand PO. Maximal oxygen uptake in athletes. J Appl Physiol. 1967;23:353-\&.

2. Dearwater SR, Laporte RE, Robertson RJ, Brenes G, Adams LL, Becker D. Activity in the spinal cord-injured patient - an epidemiologic analysis of metabolic parameters. Med Sci Sports Exerc. 1986;18:541-4.

3. Jacobs PL, Nash MS. Exercise recommendations for individuals with spinal cord injury. Sports Med. 2004;34:727-51.

4. Berlowitz DJ, Wadsworth B, Ross J. Respiratory problems and management in people with spinal cord injury. Breathe. 2016;12:328-40.

5. Wecht JM, Harel NY, Guest J, Kirshblum SC, Forrest GF, Bloom O. et al. Cardiovascular autonomic dysfunction in spinal cord injury: epidemiology, diagnosis, and management. Semin Neurol. 2020;40:550-9.

6. Partida E, Mironets E, Hou S, Tom VJ. Cardiovascular dysfunction following spinal cord injury. Neural Regen Res. 2016;11:189-94.

7. Popa C, Popa F, Grigorean VT, Onose G, Sandu AM, Popescu M, et al. Vascular dysfunctions following spinal cord injury. J Med Life. 2010;3:275-85.

8. Nash MS, Bilsker S, Marcillo AE, Isaac SM, Botelho LA, Klose $\mathrm{KJ}$, et al. Reversal of adaptive left-ventricular atrophy following electrically-stimulated exercise training in human tetraplegics. Paraplegia 1991;29:590-9.

9. Gater D. Spinal cord injury. In: Ehrman JK, Gordon PM, Visich PS, Keteyian SJ (eds). Clinical exercise physiology. Champaign, IL: Human Kinetics; 2003. p. 503-26.

10. Gater D. Exercise and Fitness with Spinal Cord Injury. Spinal Cord Injuries: Management and Rehabilitation. St. Louis, MO: Mosby Elsevier; 2010. p. 430-54. 
11. Noreau L, Shephard RJ, Simard C, Paré G, Pomerleau P. Relationship of impairment and functional ability to habitual activity and fitness following spinal cord injury. Int $\mathrm{J}$ Rehabil Res. 1993;16:265-75.

12. Doherty JG, Burns AS, O'Ferrall DM, Ditunno JF Jr. Prevalence of upper motor neuron vs lower motor neuron lesions in complete lower thoracic and lumbar spinal cord injuries. J Spinal Cord Med. 2002;25:289-92.

13. Gorgey AS, Dudley GA. Spasticity may defend skeletal muscle size and composition after incomplete spinal cord injury. Spinal Cord. 2008;46:96-102.

14. Chandrasekaran S, Davis J, Bersch I, Goldberg G, Gorgey AS. Electrical stimulation and denervated muscles after spinal cord injury. Neural Regen Res. 2020;15:1397-407.

15. Gorgey AS, Dolbow DR, Dolbow JD, Khalil RK, Gater DR. The effects of electrical stimulation on body composition and metabolic profile after spinal cord injury-Part II. J Spinal Cord Med. 2015;38:23-37.

16. Standring $S$.The anatomy of the peripheral nervous system. In: Standring S, editor. Gray's Anatomy: The Anatomical Basis of Clinical Practice. 42 edn. Edinburgh: Elsevier; 2021. p. 1464. e11-.e55.

17. Standring S. Pelvic girdle and lower limb: Overview and surface anatomy. In: Standring S, editor. Gray's Anatomy: The Anatomical Basis of Clinical Practice. 42 ed. Edinburgh: Elsevier; 2021. p. 1333-51.e2.

18. Gass GC, Camp EM. Effects of prolonged exercise in highly trained traumatic paraplegic men. J Appl Physiol. 1987;63:1846-52.

19. Spinal Cord Injury Facts and Figures at a Glance. National Spinal Cord Injury Statistical Center; 2019.

20. Farkas GJ, Gater DR. Energy expenditure and nutrition in neurogenic obesity following spinal cord injury. J Phys Med Rehabilitation. 2020;2:11-3.

21. Farkas GJ, Pitot MA, Gater DR Jr. A systematic review of the accuracy of estimated and measured resting metabolic rate in chronic spinal cord injury. Int J sport Nutr Exerc Metab. 2019;29:548-58.

22. Farkas GJ, Gorgey AS, Dolbow DR, Berg AS, Gater DR. Caloric intake relative to total daily energy expenditure using a spinal cord injury-specific correction factor: an analysis by level of injury. Am J Phys Med Rehabil. 2019;98:947-52.

23. Buchholz AC, McGillivray CF, Pencharz PB. Physical activity levels are low in free-living adults with chronic paraplegia. Obes Res. 2003;11:563-70.

24. Farkas GJ, Gater DR. Neurogenic obesity and systemic inflammation following spinal cord injury: a review. J Spinal Cord Med. 2018;41:378-87.

25. Consultation WHO. Obesity: Preventing and managing the global epidemic. Obesity: Preventing and Managing the Global Epidemic: Report of a Who Consultation. WHO Technical Report Series. 894. Geneva: World Health Organization; 2000. p. 1-253.

26. Nightingale TE, Williams S, Thompson D, Bilzon JLJ. Energy balance components in persons with paraplegia: daily variation and appropriate measurement duration. Int J Behav Nutr Phys Act. 2017;14:132.

27. Schuld C, Franz S, Bruggemann K, Heutehaus L, Weidner N, Kirshblum SC, et al. International standards for neurological classification of spinal cord injury: impact of the revised worksheet (revision 02/13) on classification performance. J Spinal Cord Med. 2016:1-9.

28. Brawner CA, Ehrman JK. Clinical exercise testing and interpretation. In: Riebe D, editor. ACSM Guidelines for Exercise Testing and Prescription. 10 edn. Philadelphia, PA: Wolters Kluwer; 2018.

29. Long CL, Schaffel N, Geiger JW, Schiller WR, Blakemore WS. Metabolic response to injury and illness: estimation of energy and protein needs from indirect calorimetry and nitrogen balance. J Parenter Enter Nutr. 1979;3:452-6.
30. Jacobs K, Gretchen A, S S, Horning M, Brooks G. Fatty acid reesterification but not oxidation is increased by oral contraceptive use in women. J Appl Physiol. 2005;98:1720-31.

31. Jacobs KA, Burns P, Kressler J, Nash MS. Heavy reliance on carbohydrate across a wide range of exercise intensities during voluntary arm ergometry in persons with paraplegia. J Spinal Cord Med. 2013;36:427-35.

32. Buchholz AC, McGillivray CF, Pencharz PB. Differences in resting metabolic rate between paraplegic and able-bodied subjects are explained by differences in body composition. Am J Clin Nutr. 2003;77:371-8.

33. Ohkawara K, Ishikawa-Takata K, Park JH, Tabata I, Tanaka S. How much locomotive activity is needed for an active physical activity level: analysis of total step counts. BMC Res Notes 2011;4:512-

34. Alizadeh A, Dyck SM, Karimi-Abdolrezaee S. Traumatic spinal cord injury: an overview of pathophysiology, models and acute injury mechanisms. Front Neurol. 2019;10.

35. Holmlund T, Ekblom-Bak E, Franzen E, Hultling C, Wahman K. Energy expenditure after spinal cord injury in people with motorcomplete tetraplegia or motor-complete paraplegia. Spinal Cord. 2018;56:274-83.

36. Standring S Gray's Anatomy: The Anatomical Basis of Clinical Practice. 40 edition ed. Edinburgh: Churchill Livingstone; 2008. $1576 \mathrm{p}$.

37. Davis GM. Exercise capacity of individuals with paraplegia. Med Sci Sports Exerc. 1993;25:423-32.

38. Hopman MT, Oeseburg B, Binkhorst RA. Cardiovascular responses in paraplegic subjects during arm exercise. Eur J Appl Physiol Occup Physiol. 1992;65:73-8.

39. Hopman MTE. Circulatory responses during arm exercise in individuals with paraplegia. Int J Sports Med. 1994;15: 126-31.

40. Hopman MTE, vanAsten W, Oeseburg B. Changes in blood flow in the common femoral artery related to inactivity and muscle atrophy in individuals with long-standing paraplegia. In: Ince C, Kesecioglu J, Telci L, Akpir K, editors. Oxygen Transport to Tissue Xvii. Adv Exp Med Biol. 3881996. p. 379-83.

41. Price M. Energy expenditure and metabolism during exercise in persons with a spinal cord injury. Sports Med. 2010;40:681-96.

42. Abel T, Schneider S, Platen P, Struder HK. Performance diagnostics in handbiking during competition. Spinal Cord. 2006;44:211-6.

43. Campbell IG, Williams C, Lakomy HKA. Physiological responses of wheelchair athletes at percentages of top speed. Br J Sports Med. 1997;31:36-40.

44. Price MJ, Campbell IG. Thermoregulatory and physiological responses of wheelchair athletes to prolonged arm crank and wheelchair exercise. Int J Sports Med. 1999;20:457-63.

45. Price MJ, Campbell IG. Thermoregulatory responses of paraplegic and able-bodied athletes at rest and during prolonged upper body exercise and passive recovery. Eur J Appl Physiol Occup Physiol. 1997;76:552-60.

46. Price MJ, Campbell IG. Thermoregulatory responses of spinal cord injured and able-bodied athletes to prolonged upper body exercise and recovery. Spinal Cord. 1999;37:772-9.

47. Kressler J, Cowan RE, Ginnity K, Nash MS. Subjective measures of exercise intensity to gauge substrate partitioning in persons with paraplegia. Topics in Spinal Cord Injury. Rehabilitation. 2012;18:205-11.

48. Laporte RE, Adams LL, Savage DD, Brenes G, Dearwater S, Cook T. The spectrum of physical-activity, cardiovascular-disease and health - an epidemiologic perspective. Am J Epidemiol. 1984;120:507-17.

49. Nash MS, Gater DR Jr. Cardiometabolic disease and dysfunction following spinal cord injury: origins and guideline-based 
countermeasures. Phys Med Rehabil Clin N. Am. 2020;31: 415-36.

50. Shetty PS, Henry CJK, Black AE, Prentice AM. Energy requirements of adults: an update on basal metabolic rates (BMRs) and physical activity levels (PALs). Eur J Clin Nutr. 1996;50:S11-23.

51. Nightingale T, Walhin J, Thompson D, Bilzon J. Biomarkers of cardiometabolic health are associated with body composition characteristics but not physical activity in persons with spinal cord injury. J Spinal Cord Med. 2019;42:328-37.

52. Chun SM, Kim HR, Shin HI. Estimating the Basal metabolic rate from fat free mass in individuals with motor complete spinal cord injury. Spinal Cord. 2017;55:844-7.

53. Nightingale TE, Gorgey AS. Predicting basal metabolic rate in men with motor complete spinal cord injury. Med Sci Sports Exerc. 2018;50:1305-12.
54. Collins EG, Gater D, Kiratli BJ, Butler J, Hanson K, Langbein W. Energy cost of physical activities in persons with spinal cord injury. Med Sci Sports Exerc. 2010;42:691-700.

55. Gorgey AS, Caudill C, Sistrun S, Khalil RE, Gill R, Castillo T, et al. Frequency of dietary recalls, nutritional assessment, and body composition assessment in men with chronic spinal cord injury. Arch Phys Med Rehabil. 2015;96:1646-53.

56. Gorgey AS, Gater DR. A preliminary report on the effects of the level of spinal cord injury on the association between central adiposity and metabolic profile. PMR. 2011;3:440-6.

57. Yilmaz B, Yasar E, Goktepe AS, Onder ME, Alaca R, Yazicioglu $\mathrm{K}$, et al. The relationship between basal metabolic rate and femur bone mineral density in men with traumatic spinal cord injury. Arch Phys Med Rehabil. 2007;88:758-61. 